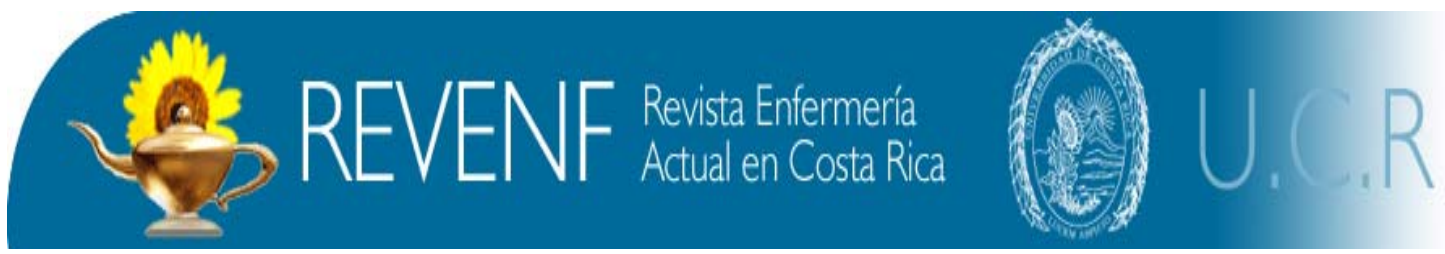

Revista Semestral Número 14 Abril - Setiembre 2008

ISSN 1409-4568

Como citar este artículo

Vega Villalobos, Nora A. Desarrollo de la Investigación en la Escuela de enfermería de la Universidad de Costa Rica. Rev. Enfermería Actual en Costa Rica [en línea].2008,No.14 [citado (fecha)]. Disponible World Wide Web: < http://www.revenf.ucr.ac.cr/articulo\%20nora.pdf $>$ ISSN 1409-4568

\title{
DESARROLLO DE LA INVESTIGACIÓN EN LA ESCUELA DE ENFERMERÍA DE LA UNIVERSIDAD DE COSTA RICA ${ }^{1}$
}

\author{
Nora Agnes Vega Villalobos ${ }^{2}$
}

\begin{abstract}
RESUMEN
En un intento por realizar una presentación expedita del desarrollo de la investigación en la Escuela de Enfermería de la Universidad de Costa Rica se expone acerca de la sistematización de los datos (propuesta hecha por Oscar Jara). El objeto es presentar cómo ha evolucionado la investigación en la Escuela antes y después del Programa de Investigación. El incremento de trabajos de en este área siguió una modalidad acentuada en el hacer de esta rama sin una influencia determinante en el trabajo cotidiano de Enfermería. Sin embargo este aspecto está cambiando, ya que el Programa de Investigación y ésta -como método de enseñanza/aprendizaje en los módulos- ha marcado la diferencia al menos en las prácticas actuales de la Escuela de Enfermería.
\end{abstract}

Palabras clave: investigación, historia, enfermería

\section{DEVELOPMENT OF THE INVESTIGATION IN THE SCHOOL OF NURSING OF THE UNIVERSITY OF COSTA RICA}

\begin{abstract}
In an attempt for realizing a prompt presentation of the development of the investigation in the School of Nursing of the University of Costa Rica it is exposed brings over of the systematizing of the information (offer done by Oscar Jara). The object is to present how it has evolved the investigation in the School before and after the Program of Investigation. The increase of works of in this one area followed a modality accentuated in to do of this branch without a determinant influence in the daily work of Nursing. Nevertheless, this aspect is changing, since the Program of Investigation and this one - as method of learning in the modules has marked the difference at least in the current practices of the School of Nursing.
\end{abstract}

Key words: research, history, nursing.

\footnotetext{
${ }^{1}$ Fecha de recepción: Noviembre del 2007

Fecha de Aceptación: Marzo del 2008

${ }^{2}$ Profesora catedrática de la Escuela de Enfermería de la Universidad de Costa Rica. Correo electrónico: agnesv@racsa.co.cr
} 


\section{INTRODUCCIÓN}

La investigación realizada en la Universidad de Costa Rica tiene supuestos epistemológicos del positivismo, de la fenomenología, y del materialismo, por mencionar algunos. Se ha permitido la libertad ideológica, sin embargo, en el área de las Ciencias de la Salud ha prevalecido la corriente positivista; corriente que la Enfermería compartió por la naturaleza de su objeto de estudio. No obstante, al buscar su propia área del conocimiento, no pudo limitarse a ese paradigma, sino más bien exploró otros paradigmas de la investigación e intentó ajustarse a la estructura universitaria, organizada por el sistema de Programas ${ }^{3}$ y Proyectos ${ }^{4}$.

Los programas tienen presupuestos reducidos, ya que sólo requieren un coordinador y -en algunos casos- apoyo temporal, sus objetivos son amplios; en el caso de los proyectos, éstos concurren por similitud temática y obedecen a objetivos específicos distintos bastante focalizados. Generalmente, presenta un enfoque unidisciplinario y contempla la participación de un investigador (a) o bien de un grupo pequeño de ellos (as). Puede ser una actividad de mediano o largo plazo. Su duración a largo plazo permite el tratamiento profundo de un determinado problema. Las actividades a corto plazo (de uno a tres años), operan con un presupuesto determinado.

El trabajo de investigación realizado en la Escuela de Enfermería de la

\footnotetext{
${ }^{3}$ Se define como “...un esfuerzo planificado de investigación establecido alrededor de un tema o problema general, que contempla la realización de varios proyectos de investigación relacionados temáticamente" (Vicerrectoría de Investigación, 2002)

${ }^{4}$ Se define como “...la puesta en práctica o la ejecución de una propuesta de investigación que ha sido aprobada por la Vicerrectoría..." (Vicerrectoría de Investigación, 1995)
}

Universidad de Costa Rica, según su historia, muestra que desde que fue institucionalizada la formación del profesional en enfermería, siempre se ha caracterizado por ser silencioso, sustentado en otras disciplinas, con conocimiento proveniente de saberes como la química, la biología y la sociología y -debido a su poco reconocimiento- poco divulgado.

Por otro lado, el propósito de las investigaciones de estos profesionales que laboran en los servicios de salud ha sido "el hacer por las personas" como fundamento de la carrera. La profesión se concentra en las personas enfermas pues este es el deseo que nace de su vocación. La actividad consistía en el "hacer por los otros", según las normas dictadas por la medicina, la microbiología, la farmacología. La independencia como disciplina es algo que para muchos es inconcebible porque no se considera una profesión con un cuerpo propio de conocimientos científicos.

Desde finales de la década de los ochenta se inició -en forma sistematizada- la investigación como una labor docente utilizada por los estudiantes del programa de licenciatura para concluir el plan de estudios con un trabajo de investigación.

El proceso ha sido coordinado por la Comisión de Trabajos Finales de Graduación; sin embargo, no se tenían identificadas líneas de investigación que fortalecerían los diferentes énfasis ofrecidos en ese momento. De aquí surge el fenómeno de hacer réplicas de diferentes investigaciones, las cuales no permitían profundizar conocimientos previos: el resultado es que quedando la práctica individual no se integraba a nivel educativo.

El producto de la experiencia en 
investigación, no ha sido utilizado sistemáticamente como fuente bibliográfica para trabajos posteriores: se ha quedado como un esfuerzo aislado sin mecanismo de retroalimentación.

Surge, entonces, la necesidad de ordenar los datos recopilados por la Escuela de Enfermería, para poder formular e inscribir el Programa de Investigación. En un esfuerzo por recapitular esta experiencia y mostrar hasta dónde se ha avanzado, se presenta la historia recopilada hasta el 2006.

\section{MATERIALES Y MÉTODO}

Para conocer y comprender el proceso de Investigación de la Escuela utilizamos la metodología de sistematización de los datos, propuesta por Oscar Jara (1994), que parte de la propia práctica.

Como requisitos están el haber participado de la experiencia y contar con registros de la experiencia desde sus inicios en 1987 hasta el 2006. Luego fue necesario establecer el objetivo, el objeto y el eje de la sistematización:

a) Objetivo:

Organizar la información de 19952006 del proceso de Investigación de la Unidad Académica para la viabilidad o formulación del Programa de Investigación.

b) Objeto de Sistematización:

Justificar la existencia de un Programa de Investigación a partir de las investigaciones elaboradas en la Unidad Académica por estudiantes y docentes,

c) Eje de la Sistematización:

Sistematizar los temas de investigación, los tipos de investigación (modalidades), la vigencia en el tiempo y la continuidad.

Se debe identificar la experiencia vivida de los proyectos y su correspondencia con los temas de investigación.

La obtención y análisis de la información se inició con la reconstrucción histórica, el ordenamiento y clasificación de la misma.

Primeramente se revisó toda la documentación; luego, se aplicaron entrevistas estructuradas a los diferentes equipos de trabajo (conformados por las docentes de la Escuela de Enfermería). Con las entrevistas se intentaba explorar diversos factores que pudieran intervenir en las experiencias de investigación -tanto personales como docentes- en áreas académicas como las licenciaturas, los módulos de Enfermería, el plan de estudios, el trabajo comunal universitario, las maestrías, la revista y la formulación de proyectos.

Para cada una de las áreas antes mencionadas se plantearon preguntas sobre la temática en estudio y se establecieron relaciones entre sí. A partir de la información recopilada, se elaboraron tablas estadísticas que sustentan el análisis y la discusión respectiva.

En relación con las limitaciones para la recopilación y ordenamiento de los datos, se encontró que el proceso ha tenido sub-registros debido a la ausencia de una base de datos.

Finalmente, se tomó en cuenta, como consideración ética, comentar los resultados de manera más objetiva, sin omitir detalles, para poder avanzar en la evolución de dicho programa.

\section{ANÁLISIS DE RESULTADOS}

El punto de partida del trabajo abarca desde la búsqueda de la documentación hasta el planteamiento de las preguntas iniciales que permitieron la $\underline{\text { Reconstrucción de la historia }}$

La investigación en la Escuela de Enfermería interesó a partir de la creación de las licenciaturas; primero 
fue de carácter general, luego con énfasis en Salud de la Mujer y Perinatología y Salud Mental y Psiquiatría. En el 2001 el mismo interés nació con las maestrías.

Estas experiencias provocaron aversión en los bachilleres de enfermería respecto del grado de licenciatura. Lo anterior tuvo dos repercusiones: primeramente, la solicitud de graduación por sala cuarta de las enfermeras diplomadas (aludiendo poseer un determinado número de créditos), y luego, la creación de escuelas de enfermería en universidades privadas para evitar la experiencia investigativa.

Las modalidades desarrolladas, en conocimiento personal y el ético. primer lugar, son las prácticas dirigidas; al segundo lugar pertenecen las tesis y seminarios de graduación, combinación de ambas modalidades y que cuentan con un número mayor de objetivos o personas que realizan el trabajo (Comisión de Trabajos Finales de Graduación, 2003).

Para resumir la cantidad de trabajos realizados, fue necesario agruparlos desde "el hacer y el ser de enfermería", tal como lo indica Carper (1978). Para el hacer se toma el conocimiento empírico dado por la cantidad de trabajos que forman parte del conocimiento estético $\mathrm{o}$ arte de enfermería; y en el ser de enfermería se incluyen

En las siguientes tablas queda ejemplificado lo anterior.

\section{Tabla 1}

Escuela de Enfermería, UCR: Distribución de trabajos finales de graduación según el conocimiento estético como campo de acción y tipo de trabajo. San Pedro, 2006

\begin{tabular}{ccccccc}
\hline Campo/tipo & $\begin{array}{c}\text { Práctica } \\
\text { dirigida }\end{array}$ & Tesis & Seminarios & Proyectos & Total \\
\hline Niñez & 41 & 6 & 4 & - & 51 \\
Adolescencia & 22 & 3 & 3 & - & 28 \\
Adultos & 78 & 15 & 4 & - & 97 \\
Adulto mayor & 21 & 4 & 2 & 1 & 28 \\
Salud de la mujer & 107 & 26 & 17 & 2 & 152 \\
y Perinatología & & & & & \\
Gerencia & 11 & 4 & 2 & - & 17 \\
Educación & 16 & 1 & 1 & - & 18 \\
Procedimientos & 17 & 2 & 5 & - & 24 \\
\hline Total & 313 & 61 & 38 & - & 415 \\
\hline
\end{tabular}

Fuente: Biblioteca de la Salud, U.C.R. 


\section{Tabla 2}

Escuela de Enfermería, UCR: Distribución de trabajos finales de graduación según el conocimiento personal como campo de acción y tipo de trabajo.

San Pedro, 2006

\begin{tabular}{ccccccc}
\hline Campo/tipo & $\begin{array}{c}\text { Práctica } \\
\text { dirigida }\end{array}$ & Tesis & Seminarios & Proyectos & Total \\
\hline Niñez & 6 & 2 & - & - & 8 \\
Adolescencia & 8 & 1 & - & - & 9 \\
Adulto & 23 & 9 & 2 & - & 34 \\
Adulto mayor & 1 & - & - & - & 1 \\
Salud mental & 17 & 4 & 4 & - & 24 \\
Educación & 3 & - & - & - & 3 \\
Procedimientos & 6 & 1 & - & - & 7 \\
\hline Total & 64 & 17 & 6 & - & 87 \\
\hline
\end{tabular}

Fuente: Biblioteca de la Salud, U.C.R.

La tabla 3 corresponde a la cantidad de trabajos de investigación hechos por los graduados.

\section{Tabla 3}

Escuela de Enfermería: Distribución de trabajos de investigación por áreas según número de graduados en quinquenios. San Pedro, 2006.

\begin{tabular}{|c|c|c|c|c|c|}
\hline \multirow[b]{2}{*}{ Áreas/años } & \multicolumn{5}{|c|}{ No. Graduados (as) } \\
\hline & $1986-1990$ & 1991-1995 & $1996-2000$ & 2001-2005 & Total \\
\hline $\begin{array}{c}\text { Licenciatura } \\
\text { General }\end{array}$ & 72 & 105 & 100 & 78 & 355 \\
\hline $\begin{array}{l}\text { Salud de la } \\
\text { mujer y }\end{array}$ & - & 96 & 183 & 38 & 317 \\
\hline Perinatología & & & & & \\
\hline Licenciatura & & & & & \\
\hline $\begin{array}{c}\text { Salud } \\
\text { Mental y }\end{array}$ & - & 44 & 43 & 41 & 128 \\
\hline Psiquiatría & & & & & \\
\hline $\begin{array}{c}\text { Maestría en } \\
\text { Salud } \\
\text { laboral }\end{array}$ & & & & 5 & 5 \\
\hline Maestría & & & & & \\
\hline $\begin{array}{l}\text { Ginecológica, } \\
\text { Obstétrica y }\end{array}$ & - & - & - & 12 & 12 \\
\hline $\begin{array}{l}\text { Perinatal } \\
\text { Maestría }\end{array}$ & & & & & \\
\hline Académica & - & - & - & 7 & 7 \\
\hline Total & 72 & 245 & 326 & 181 & 824 \\
\hline
\end{tabular}

Fuente: Libro de actas de graduación de la Escuela de Enfermería, UCR. 
La tabla cuatro presenta las condiciones de los proyectos de investigación.

Tabla 4

Escuela de Enfermería, UCR: Distribución de proyectos de investigación según condición. San Pedro, 2006.

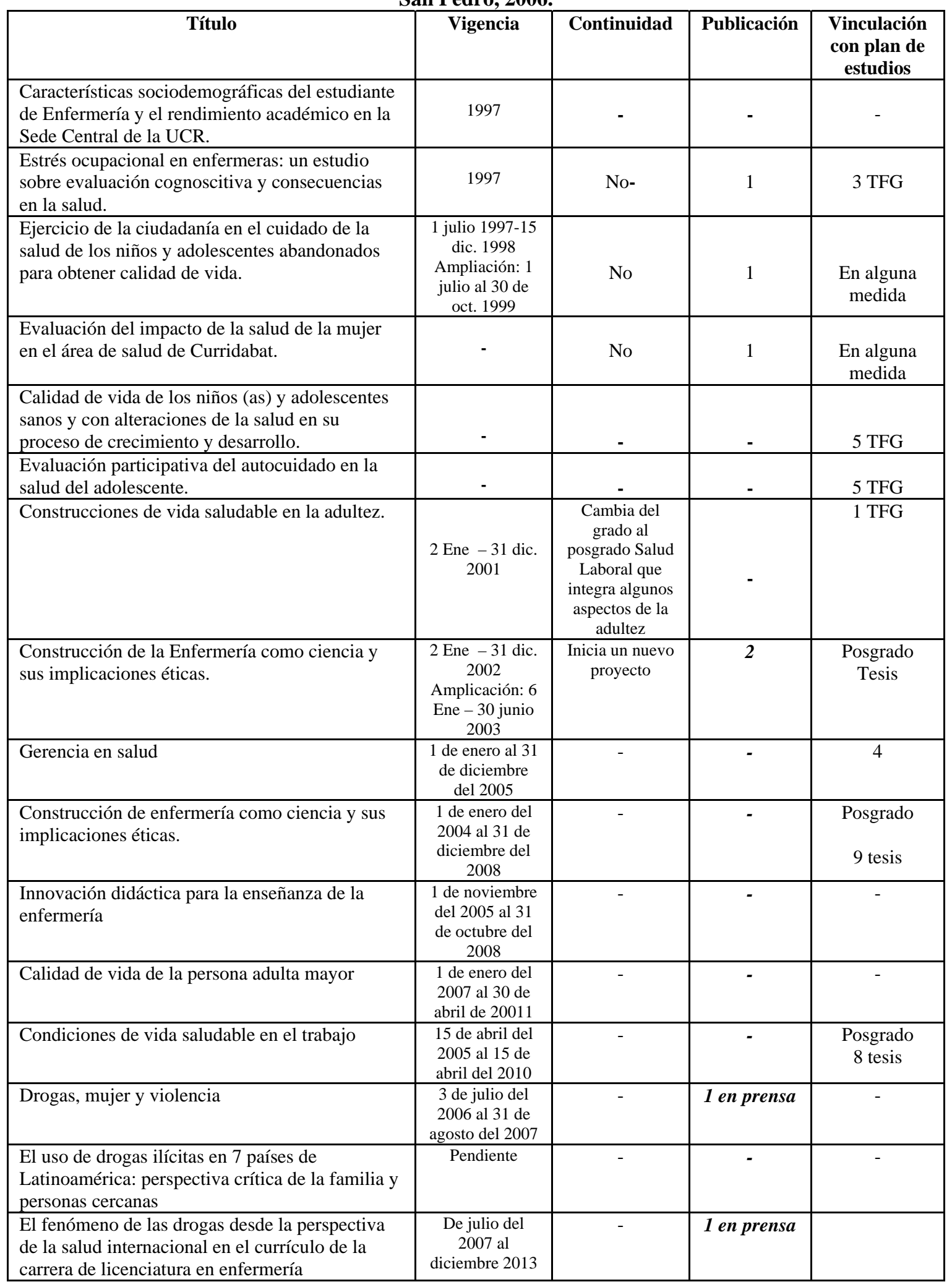


En relación con el conocimiento ético, se han realizado cuatro trabajos con la temática del adulto: una tesis y tres seminarios (Biblioteca de Salud, 2006).

Esta información merece ser revisada profundamente pues -por el título de los temas- parece que se da con mucha frecuencia una réplica del tema en diferentes escenarios. El paradigma de investigación de las tesis es el positivista y no demuestra continuidad en algún resultado. Sin embargo, no se puede afirmar certeramente, debido a que la profundización del análisis no fue minuciosa.

Los estudiantes que han estudiado con el nuevo Plan de Estudios (1998) integran la investigación como parte inherente de su hacer cotidiano, situación que ha dado un viraje importante y positivo a la profesión. (Coordinadora Comité de Currículo, 2003)

A partir del 2000 con la apertura de las maestrías, primero la Académica y luego la de Ginecología, Obstetricia y Perinatología, Enfermería Laboral, Salud Mental, y Pediatría se inició el proceso de diferenciación de nivel en los procesos investigativos. (Biblioteca de Salud, 2006)

También en los últimos diez años se han aprobado en la Vicerrectoría de Investigación proyectos de investigación de las docentes en los que se han aplicado nuevos paradigmas de investigación, medida que está cambiando la historia de Enfermería. (Comisión de Investigación, 2006)

Marca la diferencia también, el inicio, a partir del año 2001, de la revista electrónica "Enfermería Actual en Costa Rica (REVENF)", que ha permitido la divulgación del conocimiento de forma más organizada.

Al mismo tiempo, la participación de la Asamblea de Escuela en varios talleres de reflexión sobre el rumbo, las fortalezas y debilidades de la investigación en la cotidianeidad del docente así como la Jornada de Investigación (en octubre del 2002) representan intromisiones de gran peso e impacto positivo en el área de la docencia. En dicha jornada -realizada el 29 y 30 de octubre del 2002- quedó en evidencia el gran potencial existente en la disciplina, el cual no es complemento del trabajo cotidiano

En la II jornada, realizada 4, 5 y 6 de octubre del 2004, los participantes consideraron que los temas discutidos no habían sido explorados anteriormente.

La planificación de la III Jornada de Investigación demuestra el esfuerzo que se está haciendo por cumplir las metas de los objetivos contemplados por el Programa de Investigación tales como el apoyo a la Docencia, la Acción Social y la Investigación. Lo anterior significa -en un futuro- la realización de talleres y congresos cuyos temas competan a la profesión

\section{DISCUSIÓN}

La investigación en las licenciaturas (Comisión de Trabajos Finales de Graduación, 2003) ha sido un requisito para graduarse. Lo anterior es considerado, por parte de los estudiantes, un obstáculo que ha originado la apertura de licenciaturas en universidades privadas que gradúan con pruebas de grado.

La formación de los docentes respecto de dirigir los trabajos fue casi nula pues ellos mismos guiaron su trabajo de graduación. La formación que daba la licenciatura fue más teórica y algunos trabajos fueron un éxito. Cabe recalcar que la experiencia investigativa del estudiante -antes de este trabajo de graduación- era prácticamente nula 
A partir de la investigación en el Plan de Estudios (Comisión de Currículo, 2003), es imperante integrar nuevos contenidos, cambiar las estrategias de trabajo, realizar pasantías por otras instituciones y fortalecer el proceso de evaluación. El cambio en los contenidos de los cursos se dio en función de los adelantos tecnológicos y del tratamiento de las enfermedades.

La investigación en los módulos es parte del proceso de enseñanza y de evaluación pero como algo momentáneo en cada ciclo. No aparecen en el programa, razón que invita a trabajar al respecto.

La iniciativa del nuevo Plan de Estudios (desde 1998) de tener la investigación como eje transversal del curriculum dio frutos al inicio, con proyectos derivados de los módulos tales como "Calidad de vida en la niñez y adolescencia", "Autocuidado en la adolescencia" y "Calidad de vida en la adultez". Sin embargo no se consolidaron como proyectos a largo plazo; pues no se han vuelto a inscribir. A partir de un "Taller de reflexión” (2006) del módulo de adultez sana se discutió la necesidad de investigar en el aula las experiencias prácticas como el modo más eficaz de aprender haciendo. Es apenas un intento de oficializar un proceso que se ha venido haciendo sin conciencia de que significa su ejecución cotidiana. Actualmente sólo está aprobado el proyecto "Calidad de vida en el adulto mayor”.

Aunque está calando el lema de "investigar para enseñar y de enseñar para investigar", parece que las metas propuestas no han sido retomadas.

Las transformaciones citadas se han dado al convertir en una fortaleza el placer por investigar, como se ha logrado en el nuevo plan de estudios de la Escuela en el que los contenidos desarrollados varían según el resultado de las investigaciones. El cambio debe darse en la medida de que se utilice lo producido como una base para progresar.

La investigación en los módulos de Enfermería, según las coordinadoras (2003,) se ha convertido en parte del hacer de enfermería. Los temas están relacionados con el contenido de los cursos. La modalidad de investigación más usada ha sido la cualitativa (parte del segundo curso de investigación) lo que demuestra un cambio de paradigma en la profesión.

La continuidad en la forma de investigar se ha dado con ciertas dificultades debido a la necesidad de capacitación los docentes por las deficiencias de su propia formación en el grado ya que la discusión no se materializaba en acciones aplicables en la cotidianeidad. Por tanto el proceso no se interiorizó. Además, no hay involucramiento en proyectos de investigación por las creencias que hay al respecto y por la resistencia de algunas docentes a trabajar extra sin que haya verdadera disposición y mística. Siempre se busca una remuneración que no se da y menos en la medida del esfuerzo aplicado. Además, supone una exposición a críticas sobre el trabajo, tema o métodos, lo cual debilita la autoestima. La ausencia de un verdadero compromiso con el desarrollo de la profesión provoca que se hayan generado pocas publicaciones precisamente por los obstáculos mencionados.

Una experiencia adicional en el Plan de Estudios -y posterior a los módulos- ha sido la formulación de proyectos (Práctica de Enfermería, 2003) cuya formación ha sido nula en grado; en posgrado se inició con la maestría en 
Pediatría. La formulación de proyectos es un campo emergente para enfermería. La independencia de esta rama no ha sido fácil, pues es una profesión de carácter asistencial, en la que el paradigma predominante depende del paso que marque la institución empleadora. La profesión se ha desenvuelto de modo introvertido aduciendo cuestiones de género, de poder y hasta de lástima. La lucha ha sido silenciosa, se encuentra "en espera" y carece de preparación en el campo.

Lo anterior ubica a la profesión, por un lado, en una posición cómoda porque no hay de que "preocuparse" de los cambios en el sistema de Salud; pero, por otro lado, la mantiene subordinada. Además, ha existido un temor de aventurarse en propuestas para el sector externo: primero por desconocimiento de cómo hacerlas; segundo; por miedo al compromiso y, tercero, para no exponerse a la crítica.

La necesidad de desarrollar esta habilidad profesional surge en casi todas las disciplinas como respuesta a las diferentes transformaciones sociales, tales como los procesos globalizadores, la modernización del estado y la reestructuración del sector salud. Como resultado, las diferentes disciplinas han ampliado su accionar con un ejercicio profesional más autónomo, es decir, menos ligado a las instituciones estatales.

Enfoquémonos ahora en la investigación en acción social con los Trabajos Comunales Universitarios y la Extensión Docente. El Trabajo Comunal Universitario (TCU) parte de una investigación de las necesidades y problemas de la comunidad: se interviene en ésta, se ejecutan las medidas del caso $\mathrm{y}$, finalmente, es evaluada. Las modalidades de esta práctica son la sistematización y la investigación-acción para obtener soluciones a los problemas.

Aplicada en los Trabajos Comunales Universitarios, según la Comisión de Acción Social (2003), es definida como la estrategia para que los estudiantes compartan con la comunidad una parte de los beneficios obtenidos durante su formación académica, la cual ayuda a sensibilizar al nuevo profesional.

Los trabajos comunales son un requisito para graduarse en la Universidad de Costa Rica, sin embargo, han sido implementados en algunas universidades privadas. La labor no se detiene pues el aporte de diferentes proyectos ha sido bastante enriquecedor por su visión interdisciplinaria.

Los temas están relacionados, las debilidades detectadas, por ejemplo el menor con enfermedades crónicas y su entorno familiar; la adolescencia y la atención del ambiente en la comunidad.

Una dificultad con la organización de los Trabajos Comunales Universitarios es que no han surgido de las líneas de investigación de la Unidad Académica sino de inquietudes particulares de las profesoras que los han inscrito.

Por solicitud de las instituciones nacen los cursos de Extensión Docente, como una modalidad de Acción Social para compartir-con otros profesionales- los conocimientos y prácticas nuevas. Del proyecto de "Gerencia en Salud” (2005) surgió un curso de Extensión Docente sobre Gerencia de Enfermería que se impartió en tres hospitales.

Por lo tanto, es necesario que el cambio se dé en la medida que demuestre cómo la práctica fortalece la producción del conocimiento, replantee el valor de la profesión para la sociedad y la vincule con otras profesiones. El Programa de 
Investigación debe estimular que se consolide la Acción Social desde los módulos y desde las líneas de Investigación de la Unidad Académica de modo que la acción multidisciplinaria -vivenciada en los trabajos comunales- sea una forma de construcción del conocimiento desde la práctica.

Por su parte, en las maestrías, las coordinadoras (2003) consideran que la investigación ha evolucionado lentamente en su función formativa pues se ansía una graduación.

Es aventurado pronosticar que la investigación que se ha iniciado, pauta un antes y un ahora en la profesión. Son varios los obstáculos que lo impiden: la mediocridad y el ansia por graduarse impiden una adecuada evolución, la comercialización de las maestrías puede dar al traste con el fin último que es la formación de investigadores en los distintos espacios.

La investigación por tópicos específicos (líneas) no es un proceso que se fortalece, quizás por el desconocimiento de las oportunidades que ofrece al desarrollo de la carrera. Apenas se da un despertar en modalidades nuevas de investigación y el paradigma dominante en Ciencias de la Salud provoca temor para ser enfrentado.

Los cambios en el nivel de formación están dando los primeros frutos, pero la perseverancia en el camino es lo que va a dar la diferencia tan esperada. Las tesis "La construcción del conocimiento en enfermería: pasado, presente y futuro" (Meza, 2005); y "La reflexión ética en enfermería” (Molina, 2005), de la Maestría en Ciencias de la Enfermería, sirvieron como apoyo significativo en el Congreso de
Enfermería "El ser para poder hacer en Enfermería” del 2005.

La investigación es el motor que permite transformar la práctica de la profesión. Su uso apropiado y certero dictará las pautas para el cambio sustancial en el desarrollo del trabajo en Enfermería, y en consecuencia de la profesión, pues se dará el timón seguro que propicie los nuevos caminos.

Según la Directora de la revista (2003), ésta juega un papel importante para divulgar los resultados de la investigación, aunque su impacto no ha sido satisfactorio. Lo que se publica ha sido el resultado de algunas tesis, y otros trabajos de las investigaciones de los cursos.

El desarrollo de la revista es imprescindible para la evolución de Enfermería pero los profesionales deben incorporar la divulgación de los conocimientos como parte de su trabajo cotidiano. El proyecto de la Revista electrónica. \# VD 988 - 2001 que estaba inscrita en la Vicerrectoría de Docencia fue considerado - por las dos profesoras que lo desarrollaron- como una idea que debe pertenecer al programa de investigación. La publicación de artículos por parte de estudiantes y personal docente no es una práctica habitual ni sistematizada en la Escuela, por lo que es necesario reflexionar acerca del compromiso con la profesión desde la perspectiva grupal

En la Jornada de Investigación del 2002 (Comisión de Investigación, 2002) se presentó la revista. La Jornada de investigación fue un excelente momento para descubrir la forma de aprovechar herramientas que siempre habían estado de las que se desconoce su uso. Fue la primera experiencia del nuevo camino hacia la luz pública y el fortalecimiento 
de alianzas dentro $y$ fuera de la profesión.

Como resultados de las investigaciones, se evidencia que la mayoría de los estudios se han dirigido más al "hacer" que al "saber" o el "ser" de la enfermería. La presentación de propuestas demostró capacidad de investigación de los profesionales y la calidad del trabajo. Surgieron algunas recomendaciones como la realización de proyectos de investigación conjuntos entre docencia y servicio, la realización de investigaciones en todas las áreas del conocimiento, la búsqueda del equilibrio entre el "hacer" de enfermería y las destrezas y habilidades en diferentes procedimientos y la investigación como estrategia de trabajo.

Incluso se hizo conciencia acerca de la necesidad e importancia de investigar en la profesión, tanto en el trabajo grupal como en el interdisciplinario e interinstitucional.

Además, surge la necesidad de fortalecer la divulgación sistemática de los resultados de la investigación para mejorar la práctica profesional y para desarrollar habilidades en una nueva cultura de divulgación del conocimiento en enfermería

En la II Jornada del 2004, (Comisión de Investigación, 2004) se descubre un vacío en el ser profesional. Hubo una reflexión acerca de la identidad de la profesión. Se incursionó en el ser y hacer de Enfermería en forma científica, lo cual permitió tomar conciencia del papel de la investigación en Enfermería. Además, se manifestó el conocimiento como forma del saber que sostiene el hacer y lleva al ser en Enfermería y brindó un soporte importante al Congreso de Enfermería del 2005. Nace la necesidad de investigar de manera diferente a como se venía haciendo. Se consideró la investigación cualitativa tan enriquecedora como la cuantitativa; es valorada la importancia de las vivencias en investigación y su utilización como herramienta para hacerla creativa e innovadora.

Como respuesta a las jornadas se han organizado talleres de reflexión (Comisión de Investigación, 2006). En julio del 2005 fue impartido un taller sobre investigación- acción dictado por las profesoras Rosa Granados Font y Mabell Granados Hernández. La evaluación fue satisfactoria porque demostró la necesidad de profundizar en metodologías que fortalezcan la investigación en los módulos, en la maestría y en lo personal.

Otros aspectos importantes han surgido: la necesidad de que cada módulo proponga sus investigaciones. la capacitación al personal docente sobre modalidades y métodos de investigación, el reformular estrategias de investigación para la graduación en licenciaturas y permitir la diferencia de nivel con los proyectos de maestría.

El Programa de Investigación (20052010), vino a unificar los esfuerzos aislados que se han dado en los últimos años para desarrollar la profesión con bases sólidas. Ha permitido dar una imagen de orden, claridad, evolución, consecuencia con el mundo en que se vive y de preparación frente al porvenir Ha estimulado el proceso para salir de la introversión, la endogamia que mata cualquier profesión.

La viabilidad política es una realidad. Desde la formulación de las Políticas en el 2004, está pendiente el continuar con la meta perseguida por el programa y su discusión en la Asamblea de Profesores. El proceso se inició con dos talleres para la formulación de las políticas de investigación de la Unidad Académica. Hubo participación de enfermeras de varias instituciones de Salud, de la Junta 
Directiva del Colegio de Enfermeras y docentes de la Unidad Académica.

Esta experiencia motivó a la dirección del Colegio de Enfermeras a organizar un centro de investigación para la carrera de enfermería. Para esto se enfatizó que la investigación se realiza desde la práctica, por lo tanto se buscarán nuevas estrategias.

La viabilidad social queda demostrada con los resultados de los proyectos de investigación, los cuales originaron nuevas expectativas de cambio en diferentes espacios como en las modificaciones del plan de estudios, en los proyectos de intervención, en la práctica cotidiana de la profesión y en nuevas fuentes de trabajo.

En setiembre del 2005 el Comité de Curriculum invitó a la Comisión del Programa a discutir las estrategias de la investigación en la Unidad Académica (actividad importantísima para consolidar el trabajo de ambas comisiones).

La viabilidad económica no es -al inicio del Programa de Investigación- un obstáculo porque se ha trabajado con pocos recursos y los resultados han sido excelentes; ahora, conociendo las posibilidades dentro y fuera de la institución (por ejemplo, drogas en vinculación con la CICAD/ OEA), habrá más flexibilidad y necesidad de desarrollar este aspecto.

Las oportunidades para investigar son muchas, pues se pueden establecer relaciones de trabajo con otras disciplinas, por lo tanto, no sólo requiere de una fuerte organización de mantener la calidad de los trabajos de investigación.

El Programa está dando sus primeros frutos en al organizar las líneas de investigación. Incluso se han presentado proyectos que no han sido aprobados pero que indican el deseo de que se incluya esta herramienta.

\section{CONSIDERACIÓN FINAL}

La investigación en Enfermería se inició como un proceso de ensayo y error sin una propuesta definida a la cual dirigirse. A partir de la estructura del nuevo Plan de Estudios en 1998, se convierte en una estrategia de enseñanza-aprendizaje fundamental en los módulos, lo que provoca un cambio en la formación del nuevo profesional.

La investigación en Acción Social es el aspecto que debe ser mejor estructurado para extraer de la acción multidisciplinaria, en la comunidad, la información que fortalezca la evolución en enfermería.

Las tesis de maestría establecen los primeros escalones para integrar las líneas de investigación, sin embargo es necesaria una estructura organizativa más eficiente que permita consolidar el proceso de crecimiento permanentemente.

Por otro lado, es notable que la divulgación del conocimiento producido no haya tenido arraigo en las personas que investigan en Enfermería.

El sustento de la investigación, para Enfermería, según las profesoras, es que quienes la practican son aquellas que tienen formación, preocupación por un tema, carisma especial por el proceso y que creen que es el camino apropiado para el cambio. Las experiencias en el extranjero constituyen un aporte importante pero impera el sentimiento de que todo puede realizarse mejor mediante la investigación. También se 
cree que quienes lo hacen es porque la consideran el mejor camino para que la disciplina progrese, al involucrarse no temen ser criticados. (entrevista, 2005)

Se investiga primero como una prueba para descubrir si funciona; luego, impulsados por el hallazgo de soluciones, prueban con nuevas técnicas que podrán ser implementadas si son efectivas. La investigación sirve para modificar formas de trabajar, variar contenidos de los cursos, demostrar la razón de ser de la profesión, dar el norte al trabajo de cualquier profesión y establecer un límite entre crear y repetir lo ya creado.

Las Jornadas de investigación presentan una realidad de cómo Enfermería hace grandes esfuerzos (a pesar de la escasez de recursos) en silencio para construir una cultura que consuma el producto de las investigaciones.

Aunado a esto, la organización del Programa de Investigación ha incursionado en la ejecución de talleres de capacitación -para los investigadores- sobre diferentes metodologías. La apertura de los cursos inicia un camino nuevo que debe ser explotado y compartido con las personas de Enfermería que laboran en las diferentes instituciones de salud.

\section{BIBLIOGRAFÍA}

Biblioteca de Salud. (2006) Trabajos Finales de Graduación. Universidad de Costa Rica: Escuela de Enfermería.

Carper, Barbara A (1978) Fundamental Patterns of Knowing in Nursing. Aspen Systems Corporation. 0161-92-68: 13-23.

Comisión de Acción Social (2003) Reporte sobre los temas y proyectos de Trabajos Comunales Universitarios y cursos de Extensión Docente. Universidad de Costa Rica: Escuela de Enfermería

Comisión de Investigación (2005) Informe final del Taller de reflexión. Universidad de Costa Rica Escuela de Enfermería
Comisión de Investigación (2003) Organización del Comité de Investigación de la Unidad Académica desde junio de 1995.: Libro de Actas. Universidad de Costa Rica: Escuela de Enfermería.

Comisión de Trabajos Finales (2003) Informe de la Comisión de Trabajos Finales de Graduación de los temas y proyectos de graduación de las licenciaturas: libro de actas. Universidad de Costa Rica Escuela de Enfermería

Comité de Curriculum, Credenciales y Reconocimientos (2003) Informe de la Coordinadora sobre los cambios curriculares acontecidos por la investigación. Universidad de Costa Rica: Escuela de Enfermería

Coordinadoras de los Módulos de Enfermería (2003) Informe Universidad de Costa Rica: Escuela de Enfermería

Curso: Práctica de Enfermería. (2003) Proyecto. Universidad de Costa Rica: Escuela de Enfermería

Directora de Posgrado. (2006). Informe de los temas de las investigaciones del Programa de Posgrado en Ciencias de la Enfermería. Universidad de Costa Rica: Posgrado en Enfermería

Directora de la Revista Electrónica de Enfermería (2003). Informe. Universidad de Costa Rica: Escuela de Enfermería.

Jornada de investigación (2002) Informe final Universidad de Costa Rica Escuela de Enfermería.

Jornada de investigación (2004) Informe final Universidad de Costa Rica Escuela de Enfermería

Meza Benavides, Mary. (2005). “La construcción del conocimiento en enfermería: pasado, presente y futuro" San José: Universidad de Costa Rica (Tesis de posgrado)

Molina Juárez Xinia (2005) “La reflexión ética en enfermería” San José: Universidad de Costa Rica (Tesis de posgrado)

Murillo Castro, Ligia. (2005) Proyecto: Gerencia en Salud. Universidad de Costa Rica: Escuela de Enfermería.

Vicerrectoría de Investigación (1993) Guía para la presentación de Programas y líneas de investigación. Ciudad Universitaria Rodrigo Facio: Vicerrectoría de Investigación, 8/ 6/ 93.

Vicerrectoría de Investigación (1993) Sistema de Formulación de proyectos. Guía para la formulación, 2001/2002. 